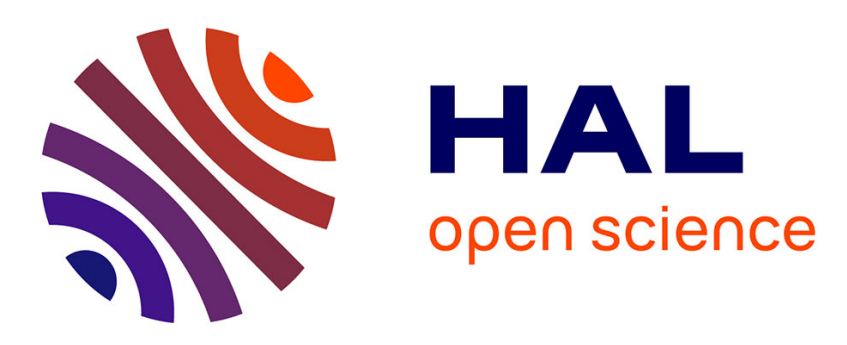

\title{
Half-optical-cycle damped solitons in quadratic nonlinear media
}

Faezeh Asadi, Babak Shokri, Hervé Leblond

\section{To cite this version:}

Faezeh Asadi, Babak Shokri, Hervé Leblond. Half-optical-cycle damped solitons in quadratic nonlinear media. Optics Communications, 2013, 294, pp.283-288. 10.1016/j.optcom.2012.12.042 . hal03204329

\section{HAL Id: hal-03204329 \\ https://univ-angers.hal.science/hal-03204329}

Submitted on 21 Apr 2021

HAL is a multi-disciplinary open access archive for the deposit and dissemination of scientific research documents, whether they are published or not. The documents may come from teaching and research institutions in France or abroad, or from public or private research centers.
L'archive ouverte pluridisciplinaire HAL, est destinée au dépôt et à la diffusion de documents scientifiques de niveau recherche, publiés ou non, émanant des établissements d'enseignement et de recherche français ou étrangers, des laboratoires publics ou privés. 


\title{
Half-optical-cycle damped solitons in quadratic nonlinear media
}

\author{
Faezeh Kimiaee Asadi ${ }^{\mathrm{a}}$, Babak Shokri ${ }^{\mathrm{a}, \mathrm{b}, *}$, Hervé Leblond ${ }^{\mathrm{c}}$ \\ a Laser-Plasma Research Institute, Shahid Beheshti University, G.C., Evin, Tehran, Iran \\ ${ }^{\mathrm{b}}$ Department of Physics, Shahid Beheshti University, G.C., Evin, Tehran, Iran \\ ' LUNAM Université, Université d'Angers, Laboratoire de Photonique d'Angers, EA 4464, 2 Bd. Lavoisier, 49045 Angers Cedex 01, France
}

\section{A R T I C L E I N F O}

Article history:

Received 31 October 2012

Received in revised form

11 December 2012

Accepted 12 December 2012

Available online 8 January 2013

Keywords:

Few-cycle pulses

Few-cycle solitons

Korteweg-de Vries equation

$\mathrm{KdV}$ equation

Korteweg-de Vries Burger equation

KdVB equation

\begin{abstract}
A B S T R A C T
In this paper, Using a classical model of the radiation-matter interaction, we show that the propagation of $(1+1)$ dimensional few-optical-cycle pulses in quadratic nonlinear media, taking moderate absorption into account, can be described by the Korteweg-de Vries-Burgers' (KdVB) equation without using the slowly varying envelope approximation. To fulfill this purpose we use the reductive perturbation method and consider the long-wave approximation, assuming that the characteristic frequency of the pulse is much lower than the resonance frequency of the atoms. We also study both analytical and numerical solution of the KdVB equation describing damped few-optical-cycle soliton propagation.
\end{abstract}

(c) 2013 Elsevier B.V. All rights reserved.

\section{Introduction}

Over the last few years, production of few-cycle pulses (FCPs) $[1-3]$ and their various applications such as material processing [4], attosecond physics [5,6], extreme nonlinear optics and light-matter interaction, have received extensive interest. Although it has been shown that theoretical models based on the slowly varying envelope approximation (SVEA), i.e., nonlinear Schrödinger type equations, are able to describe the propagation of optical pulses down to ten optical cycles in nonlinear dispersive media [7-10], the validity of this approach is contested for shorter pulses and definitely falls down in the single- and subcycle ranges. For pulses considered in the present paper with duration of only a few optical cycles, we consider a non-SVEA dynamical model in which the concept of the envelope has not been used [11-13].

Recently propagation of FCPs in nonlinear media has attracted a lot of attention and it has been shown that the physics of $(1+1)$ dimensional FCP solitons in Kerr media could be adequately described beyond the limitation of the SVEA by means of the modified Korteweg-de Vries (mKdV) $[13,14]$, the sine-Gordon (sG) [15], or mKdV-sG [16] equations. Vectorial models have also been proposed, showing the existence of circularly polarized FCP

\footnotetext{
* Corresponding author. Tel.: +9821224317 73; fax: +9821224317 75

E-mail addresses: f.kimiaee@mail.sbu.ac.ir (F. Kimiaee Asadi), babakshokri@yahoo.com, b-shokri@sbu.ac.ir (B. Shokri), herve.leblond@univ-angers.fr (H. Leblond).
}

solitons [17-19]. Dissipative FCP solitons are another subject of interest whose formation in active nonlinear optical fibers has been studied in Ref. [20] by means of the Maxwell-Bloch equations. Moreover, starting from a model of the same type, a non-SVEA version of the Lorenz-Haken equation governing the physics of dissipative FCP solitons in the laser cavity has been derived [21,22]. Propagation of ultrashort optical solitons in (conservative) quadratic media has also been considered, either by the direct numerical solution of the Maxwell-Bloch Eqs. (23) and (24) or reducing them to the Korteweg-de Vries (KdV) Eqs. (25) and (26). In both mentioned approaches [23-26], it has been shown that the FCP input evolves into unipolar half-cycle pulses.

FCP soliton propagation requires a strong nonlinear effect. Therefore, a material with a high nonlinear susceptibility is required as a propagation medium. It is but well known from the Kramers-Kronig relations [27] that, if the real part of the nonlinear susceptibility is large, the imaginary part is also large, i.e., damping occurs. On the other hand, according to Millers' rule [28], the nonlinear and linear susceptibilities are not independent, and the resonance of the fundamental frequency has an important effect on the magnitude of the second order susceptibility. Hence, although soliton propagation assumes in principle that the linear absorption can be neglected, it will very hardly be the case in a real experiment.

The aim of the present paper is to derive an equation which governs the dynamics of $(1+1)$ dimensional few-optical-cycle damped solitons in quadratic nonlinear media beyond the SVEA. For this purpose, damping is assumed to be small and treated as a 
perturbation so that the assumption made in Ref. [25] by which the central pulse frequency is far from the resonance frequency of the atoms can be used. The resonance frequency can be far below the central pulse frequency (typically in the infrared range), or far above it (typically in the ultraviolet range). The former case can be described by using a short-wave approximation and the latter, which is considered here, is treated by means of a long-wave approximation.

The present paper is organized as follows. Section 2 presents a basic set of equations which introduces our classical model. By using the reductive perturbation method in the long-wave approximation, the KdVB equation governing the propagation of $(1+1)$ dimensional damped FC solitons is derived in Section 3. Section 4 deals with both analytical and numerical solution of the KdVB equation, and finally in Section 5 the conclusion is presented.

\section{Classical model}

We consider a classical model of an elastically bounded electron oscillating perpendicular to the propagation direction of a wave $(z)$. The wave is linearly polarized along the oscillation direction $(x)$. The evolution of the position $x$ of the atomic electron is described by the anharmonic oscillator equation

$\frac{d^{2} x}{d t^{2}}+2 \Omega \tilde{\gamma} \frac{d x}{d t}+\Omega^{2} x+a x^{2}=-\frac{e}{m} E$,

where $m$ is the electron mass, $-e$ is its charge, $\Omega$ is the resonance frequency, $E$ is the electric field component along the $x$ axis and $\tilde{\gamma}$ is the damping ratio. Here, damping is assumed to be weak so that it is considered as a perturbation. Hence we set

$\tilde{\gamma}=\varepsilon^{p} \gamma$,

where $\gamma$ is a normalized damping parameter, $\varepsilon$ is a small parameter and $p$ is a positive integer.

The Maxwell equations are used to describe the evolution of $E$; in the absence of the magnetic field and in the scalar $(1+1)$ dimensional case, they reduce to

$\partial_{z}^{2} E=\frac{1}{c^{2}} \partial_{t}^{2}(E+4 \pi P)$,

where $P=-$ Nex is the polarization density and $N$ is the density of atoms.

In the most restricted sense, soliton propagation requires a conservative system, and hence no absorption or damping occurs. From the optics point of view, it means that the resonance frequency $\Omega$ of the atoms is far away enough from the characteristic frequency $\omega_{w}$ of the considered radiation, so that absorption can be neglected. In the present paper, damping is taken into account but is assumed to remain small. Mathematically, it is treated as a perturbation (Eq. (2)). Hence we can assume that $\Omega$ remains far from $\omega_{w}$. Then, the long wave approximation can be considered in which $\Omega$ is much larger than $\omega_{w}$. Since $\omega_{w}$ is assumed to belong to the visible range, $\Omega$ belongs to the ultraviolet.

The derivation is performed using the reductive perturbation method $[29,30]$. The long wave approximation involves the introduction of the slow variables

$\tau=\varepsilon\left(t-\frac{z}{V}\right), \quad \zeta=\varepsilon^{3} z$,

where $V$ is the propagation speed. The retarded time $\tau$ describes the shape of the pulse. According to the assumption $\Omega \gg \omega$, the variable $\tau$ is a slow variable, accounting for a wave period large with respect to the period corresponding to the resonance frequency $\Omega$. For a FCP, the pulse duration has the same order of magnitude as the optical period, which formally is long in this formalism. The other slow variable, $\zeta$, shows long distance propagation.

In order to have a soliton perturbed by a small amount of damping, nonlinearity and dispersion must arise simultaneously during the propagation distance. Thus, considering weak amplitude assumption and using the reductive perturbation method [30], we expand the polarization density and the electric field in power series of the small parameter $\varepsilon$ as

$P=\sum_{n>1} \varepsilon^{n} P_{n}, \quad E=\sum_{n>1} \varepsilon^{n} E_{n}$

\section{Derivation of the KdVB equation}

We give here the main lines of the derivation, further detail can be found in [25], which presents an analogous derivation in the more restricted case where damping is neglected. Substituting expressions (4) and (5) into Eqs. (1) and (3) (where $P=-N e x$ ), and considering the terms in the different powers of $\varepsilon$, Eq. (1) at the order of $\varepsilon^{2}$, results in

$P_{2}=\frac{N e^{2}}{m \Omega^{2}} E_{2}$.

Using Eqs. (6) and (3) at the order of $\varepsilon^{4}$ gives the value of the velocity as below

$V=c\left(1+\frac{4 \pi N e^{2}}{m \Omega^{2}}\right)^{-1 / 2}$.

From Eq. (1), it is seen that the lowest power of $\varepsilon$ for the damping term is $p+3$. Thus, according to different positive integer values which can be taken by $p$, two different cases arise:

Case (a): For $p=1$, From Eq. (1) at the order of $\varepsilon^{4}$, we get

$P_{4}=\frac{N e^{2}}{m \Omega^{2}} E_{4}-\frac{2 \gamma N e^{2}}{m \Omega^{3}} \partial_{\tau} E_{2}+\frac{a N e^{3}}{m^{2} \Omega^{6}}\left(E_{2}\right)^{2}-\frac{N e^{2}}{m \Omega^{4}} \partial_{\tau}^{2} E_{2}$.

Substituting Eq. (8) into Eq. (3) at the order of $\varepsilon^{6}$ and considering Eq. (7), the terms that contain $E_{4}$ cancel each other and we obtain the following KdVB equation

$\partial_{\zeta} E_{2}=A \partial_{\tau}^{3} E_{2}+B \partial_{\tau} E_{2}^{2}+C \partial_{\tau}^{2} E_{2}$,

where coefficients

$A=\frac{2 \pi V N e^{2}}{m c^{2} \Omega^{4}}, \quad B=\frac{-2 \pi V a N e^{3}}{m^{2} c^{2} \Omega^{6}}, \quad C=\frac{4 \pi V N \gamma e^{2}}{m c^{2} \Omega^{3}}$,

are related to dispersion, nonlinearity and damping, respectively.

Case (b): For $p>1$, Eq. (1) at the order of $\varepsilon^{4}$ gives

$P_{4}=\frac{N e^{2}}{m \Omega^{2}} E_{4}+\frac{a N e^{3}}{m^{2} \Omega^{6}}\left(E_{2}\right)^{2}-\frac{N e^{2}}{m \Omega^{4}} \partial_{\tau}^{2} E_{2}$.

Thus, using Eq. (7) and substituting Eq. (11) into Eq. (3) at the order of $\varepsilon^{6}$, we get

$\partial_{\zeta} E_{2}=A \partial_{\tau}^{3} E_{2}+B \partial_{\tau} E_{2}^{2}$,

which is the KdV equation that has been studied in Ref. [25]. Coefficients $A$ and $B$ demonstrating dispersion and nonlinearity, respectively, are the same as the case (a).

Formally, in the case of $p>1$, i.e., $p \geq 2$, damping effect is too weak and does not have enough time to arise so it can be neglected.

The susceptibilities pertaining to the model Eq. (1) are computed by seeking the response of the latter to a field $E=A e^{i \omega t}+c c$; we obtain

$\chi^{(1)}(\omega)=\frac{4 \pi N e^{2}}{m\left(\Omega^{2}-\omega^{2}+2 i \omega \Omega \tilde{\gamma}\right)}$, 
$\chi^{(2)}(0 ; \omega,-\omega)=\frac{4 \pi N e^{3} a}{m^{2} \Omega^{2}\left(\Omega^{2}-\omega^{2}\right)^{2}}$,

$\chi^{(2)}(2 \omega ; \omega, \omega)=\frac{4 \pi N e^{3} a}{m^{2}\left(\Omega^{2}-4 \omega^{2}\right)\left(\Omega^{2}-\omega^{2}\right)^{2}}$.

The absorption has been neglected in the expression of the nonlinear susceptibility. We see that Eq. (7) is $V=c / n$ with $n^{2}=\left(\chi^{(1)}\right)_{\omega=0}$, in which damping is neglected. Still neglecting damping, we set

$\beta_{2}=\left(\frac{d^{2} \chi^{(1)}}{d \omega^{2}}\right)_{\omega=0}=\frac{8 \pi N e^{2}}{m \Omega^{4}}$,

it is seen that the dispersion coefficient in the KdVB Eq. (9) is

$A=\frac{\beta_{2}}{4 n c}$,

according to Refs. [25,26]. In the presence of long wave approximation, $\omega \rightarrow 0$, both $\chi^{(2)}(0 ; \omega,-\omega)$ and $\chi^{(2)}(2 \omega ; \omega, \omega)$ coincide, let us denote by

$\chi^{(2)}=\frac{4 \pi N e^{3} a}{m^{2} \Omega^{6}}$,

their common value. Then the nonlinear coefficient in (9) is

$B=\frac{-1}{2 n c} \chi^{(2)}$,

as in Refs. [25,26] again. $\operatorname{Im}\left(\chi^{(1)}\right)$ is computed in the limit of both small $\gamma$ and $\omega$, as

$\operatorname{Im}\left(\chi^{(1)}\right)=\frac{-8 \pi N e^{2} \omega \tilde{\gamma}}{m \Omega^{3}}$,

and consequently, the damping coefficient in Eq. (9) is

$C=\frac{-1}{2 n c}\left(\frac{\operatorname{Im} \chi^{(1)}}{\omega}\right)_{\omega=0}$.

Since the absorption coefficient is $\alpha=-\omega \operatorname{Im} \chi^{(1)} /(n c)$, it is related to the latter through $C=\alpha / 2 \omega^{2}$, where $\omega$ is the angular frequency at which $\alpha$ has been measured.

\section{The damped soliton}

In order to find the asymptotic solution of the KdVB equation, we commence with the case of $C=0$, in which Eq. (9) reduces to Eq. (12).

It is well known that the KdV equation can be solved by means of the inverse scattering transform (IST) [31]. Moreover, $N$-soliton solutions can be obtained by different methods such as the Hirota one [32]. The fundamental soliton can also be obtained by direct calculation, derived first in 1871 by Boussinesq [33]. In the present case, the fundamental soliton is written as

$E_{2}=\frac{6 m \Omega^{4} q^{2}}{-a e} \operatorname{sech}^{2}\left[q \Omega\left(\tau+\frac{8 \pi V N e^{2}}{m c^{2} \Omega^{2}} q^{2} \zeta\right)\right]$,

where $q$ is a positive number. For the sake of simplicity, the KdV equation in its dimensionless form is written as

$\partial_{Z} U=\partial_{T}^{3} U+6 U \partial_{T} U$,

in which $U, Z$ and $T$ are dimensionless variables in the form of

$U=\frac{E_{2}}{E_{0}}, \quad Z=\frac{\zeta}{L_{0}}, \quad T=\frac{\tau}{T_{0}}$, where

$E_{0}=\frac{3 A}{B T_{0}^{2}}=\frac{-3 \beta_{2}}{2 \chi^{(2)} T_{0}^{2}}, \quad L_{0}=\frac{T_{0}^{3}}{A}=\frac{4 n c T_{0}^{3}}{\beta_{2}}$,

And $T_{0}$ is arbitrary.

Using values pertaining to beta barium borate (BBO) (extraordinary index) given by textbooks [34] and industrials [35], and taking as a reference time $T_{0}=0.8 \mathrm{fs}$, so that a dimensionless angular frequency $\omega=1.5$ corresponds to a wavelength very close to $1 \mu \mathrm{m}$, we get $E_{0}=9.6 \times 10^{9} \mathrm{~V} / \mathrm{m}$ and $L_{0}=53 \mu \mathrm{m}$. The onesoliton solution of the KdV equation is shown in Fig. 1.

The normalized form of the $\operatorname{KdVB}$ equation $(C \neq 0)$

$\partial_{Z} U=\partial_{T}^{3} U+6 U \partial_{T} U+2 \Gamma \partial_{T}^{2} U$

with $\Gamma=\tilde{\gamma} \Omega T_{0}$, is obtained by using the same linear transformation as the $\mathrm{KdV}$ equation without changing the propagation direction. Using again data pertaining to BBO [35], it is said that the absorption coefficient $\alpha<0.1 \% / \mathrm{cm}$. Let us assume $\alpha$ equal to this maximal value; then, using Eqs. (20) and (21), with $\omega$ corresponding to the wavelength $1 \mu \mathrm{m}$ at which $\alpha$ was measured, we get $\tilde{\gamma} \simeq 6 \times 10^{-8}$. Further, $\Omega$ is evaluated in such a way that the value of $\beta_{2}$ computed according to Eq. (16) coincides with the measured value, i.e., $\Omega=\sqrt{2\left(n^{2}-1\right) / \beta_{2}}$. Still for BBO, extraordinary index, we get $\Omega=12.4 \mathrm{rad} / \mathrm{fs}$, and consequently $\Gamma \simeq 6 \times 10^{-7}$. It is seen that the perturbative approach is fully justified, one may even consider that linear absorption is too small to be taken into account. Much higher values can be reached in other materials, for example, in $\mathrm{ZnGeP}_{2}$ at $1.2 \mu \mathrm{m}$ [36], $\alpha$ is about $1 / \mathrm{cm}$, we get $\tilde{\gamma} \simeq 10^{-4}, \Omega=2.5 \mathrm{rad} / \mathrm{fs}$ and $\Gamma \simeq 2 \times 10^{-4}$. Much closer to the resonances, dissipation becomes much higher, however dispersion increases in the same time, and our derivation of the KdVB model ceases to be valid, which prevents us to give quantitative data in this domain. However, second harmonic generation in the UV domain has been considered in such materials [37]. Hence we consider below large values of tilde gamma, much higher than experimentally available, we show that: even in this range of huge absorption, linear absorption does not prevent $\mathrm{KdV}$ soliton formation.

An approximate analytical expression of the damped soliton solution can be obtained by using the Krylov-Bogoliubov expansion, which has already been done by Ott and Sudan [38,39]. Considering

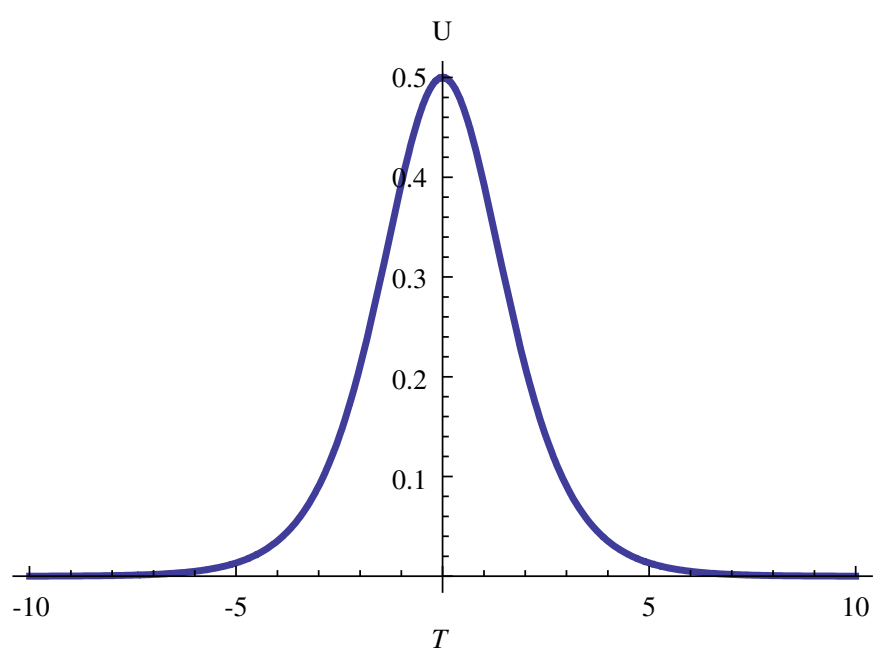

Fig. 1. The FCP soliton in quadratic nonlinear media (Soliton solution to KdV). 
Eqs. (9) and (10), this approximate analytical expression yields

$$
E_{2} \approx-K(\zeta) \operatorname{sech}^{2}\left[\sqrt{\frac{a e K(\zeta)}{6 m \Omega^{2}}}\left(\tau-\tau_{0}+\frac{4 \pi V a N e^{3}}{3 m^{2} c^{2} \Omega^{6}} \int_{0}^{\zeta} K(\zeta) d \zeta\right)\right],
$$

where

$$
K(\zeta)=\frac{K_{0}}{1+\zeta / \zeta_{0}}
$$

with

$$
\zeta_{0}=\frac{45 m^{2} c^{2} \Omega^{5}}{32 K_{0} \pi V a N e^{3} \gamma}
$$

is the amplitude of the damped soliton and $K_{0}=6 m \Omega^{4} q^{2} / a e$ is the amplitude of the KdV soliton obtained from Eq. (22). Obviously, $\int_{0}^{\zeta} K(\zeta) d \zeta=K_{0} \zeta_{0} \ln \left(1+\left(\zeta / \zeta_{0}\right)\right)$. The evolution of the damped KdVB soliton for some rather high values of the damping ratio is shown in Fig. 2.

It should be noted that Eqs. (22) and (27) are written by weak amplitude and damping assumptions in a frame traveling at the group velocity of the linear wave. Thus, by getting back to the laboratory variables the electric field would be $\tilde{E}=\varepsilon^{2} E_{2}$, and since $K_{0}$ is the maximum of $E_{2}$ at $\zeta=0$ it is considered as $\tilde{K}_{0}=\varepsilon^{2} K_{0}$. Comparing Eqs. (22) and (27) in the laboratory variables, one can see that in the presence of damping, the amplitude of the solitary wave decreases according to

$\tilde{K}(z)=\frac{\tilde{K}_{0}}{1+z / z_{0}}$
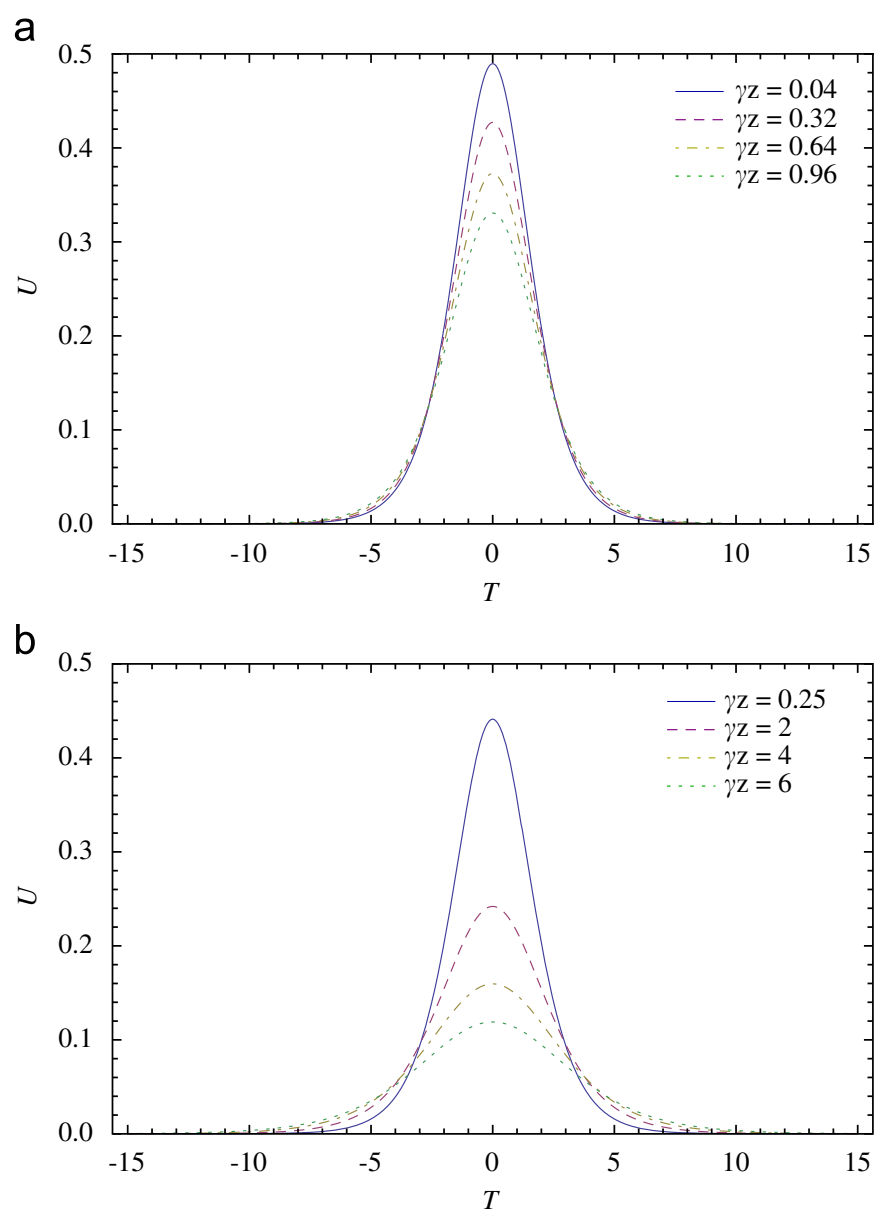

Fig. 2. Evolution of the damped solitons for different propagation distances. (a) for $\Gamma=0.008$ and (b) for $\Gamma=0.05$. with $z_{0}=-45 / 16 \tilde{K}_{0} \tilde{\gamma} B \Omega=45 n c / 8 \chi^{(2)} \tilde{K}_{0} \tilde{\gamma} \Omega$, where $\tilde{\gamma}=\varepsilon \gamma$, and therefore its corresponding width increases. This process is accompanied by the decrease of the propagation speed according to

$v=V\left(1-\frac{2 V \tilde{K}_{0} B}{3\left(1+\frac{16 \tilde{K}_{0} \tilde{\gamma} B \Omega}{45} z\right)}\right)^{-1}$,

in which $B$ is the nonlinear coefficient obtained from Eq. (10). In Eq. (31) it is seen that $v$ decreases monotonically from $v=V /\left(1-2 V \tilde{K}_{0} B / 3\right)$ at $z=0$, to $V=c / n$ as $z \rightarrow \infty$. The damping distance is evaluated according to $z_{0}=15 \tau^{2} /(16 C)=15 \omega^{2} \tau^{2} /(8 \alpha)$. Assuming the values obtained above for BBO and a very short KdV soliton of only $1 \mathrm{fs}$ duration, we get $z_{0} \simeq 67 \mathrm{~m}$. It is seen that in this case damping is completely negligible, even more than for linear waves. Using value pertaining to $\mathrm{ZnGeP}_{2}$ at $1.2 \mu \mathrm{m}, \alpha=1 / \mathrm{cm}$, again for a $1 \mathrm{fs}$ pulse, we obtain $z_{0}=4.6 \mathrm{~cm}$.

The general theory of the IST states that, within the KdV model, any input is expected to evolve into a set of solitons and 'radiation', i.e., dispersive waves [31]. This issue has been discussed in detail in Refs. $[25,26]$ for the case of FCP-type inputs, with the form of a Gaussian envelope modulating a cosine. Here the question is whether this evolution of a FCP toward one or a few solitons still will occur in the presence of damping or not, especially in the case of very high damping. This case can be discussed by a numerical approach. The KdVB Eq. (26) is solved numerically using the exponential time differencing method [40], in which the modified Euler scheme is used. We use a specific choice of the FCP input, for which a single soliton and reasonably a little radiation is produced in the absence of damping.

In particular, when the input is $U=10 \cos (1.5 T) \operatorname{sech}^{2}(3 T / 8)$, the result of the evolution for several values of the damping constant $\Gamma$ is shown in Fig. 3. One can observe that for small damping, the soliton is slightly modified. However, for larger damping (e.g., $\Gamma \sim 1$ for the considered value of parameters), the damped soliton and the wave profile resulting from the damping of the radiative wave have comparable amplitudes. In the intermediate range, as shown in Fig. 4, damped solitons are formed. It is worth mentioning that similar to the conservative case [25], the generation of solitons depends on the carrier-envelope phase of the input FCP. As an example, for a carrier-envelope phase of $\pi$, in the conservative case two solitons with equal amplitudes are emitted. However, in our case the slowest soliton which is closer to the radiative waves,
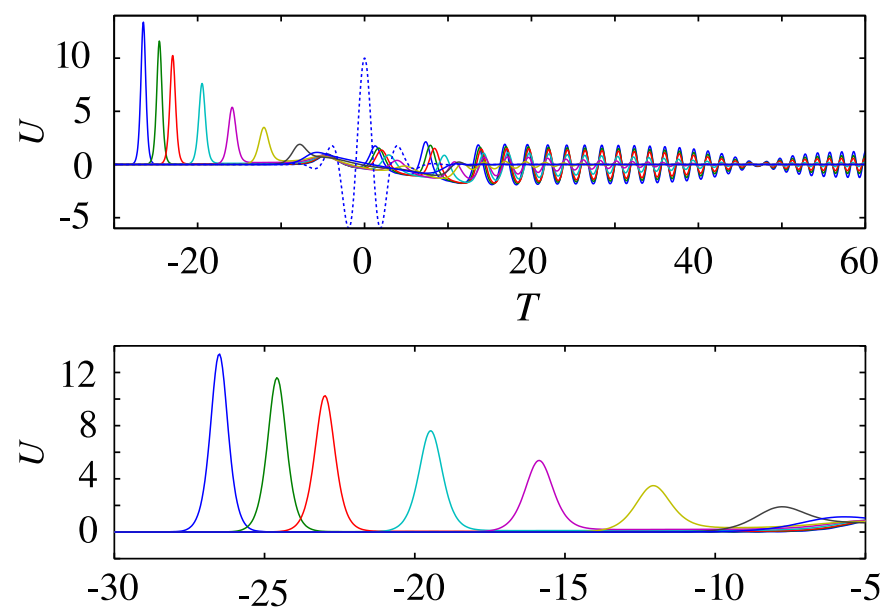

Fig. 3. Evolution of a FCP input (dashed line) into a KdV soliton (solid lines) and radiation in the presence of damping, for several values of the damping constant $\Gamma$. The bottom figure is a zoom of the top one showing the produced damped solitons only. The leftmost soliton with highest amplitude is obtained for $\Gamma=0$, the following ones for $\Gamma=0.01,0.02,0.05,0.1,0.2,0.5$, and 1 . The propagation distance is $Z=1$. 


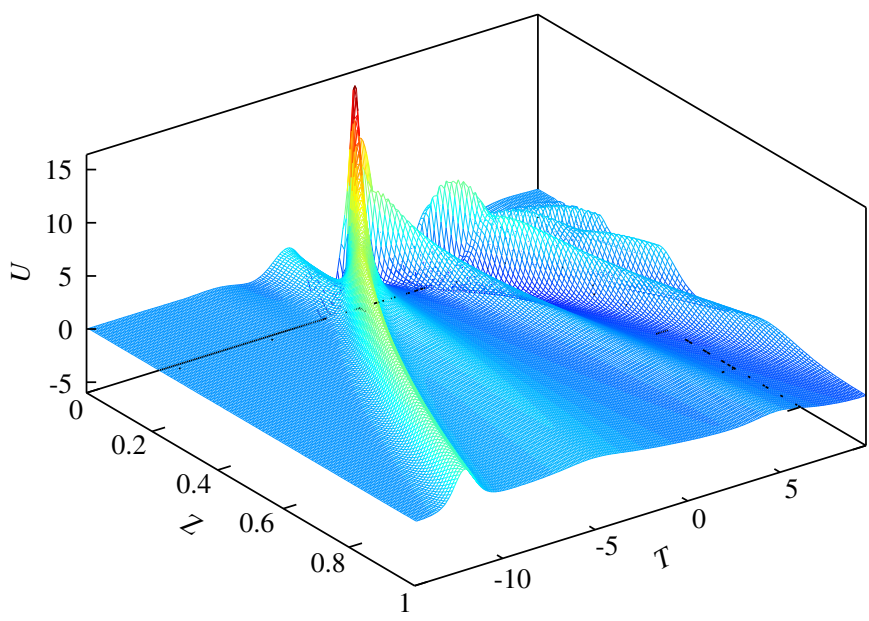

Fig. 4. Evolution of a FCP input into a damped soliton. Parameters are the same as in Fig. 3 with $\Gamma=0.2$.

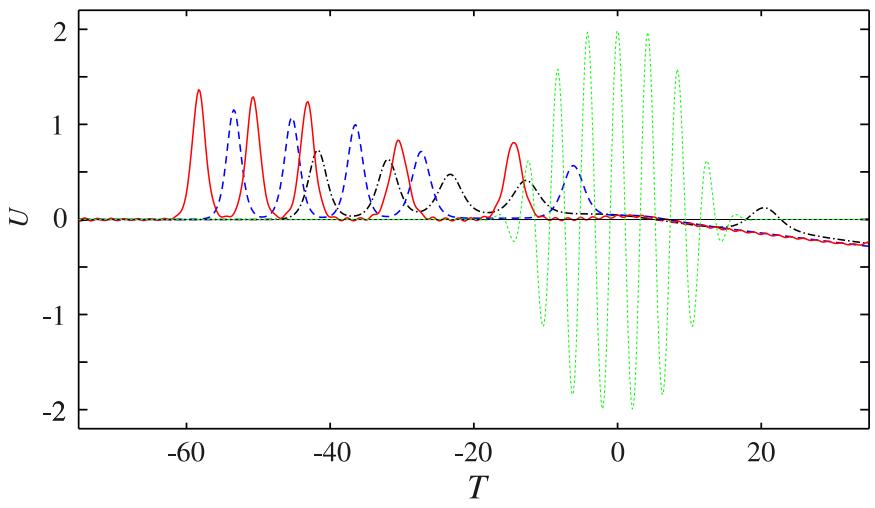

Fig. 5. Formation of several solitons from a FCP, in the presence of damping. The dotted green line shows the input, and the other lines the soliton part of the output after a normalized propagation distance $Z=20$, for various values of the damping constant. The red solid: $\Gamma=0$, blue dashed: $\Gamma=0.005$, black dashdotted: $\Gamma=0.025$. (For interpretation of the references to colour in this figure legend, the reader is referred to the web version of this article.)

suffers stronger damping than the fastest one, which breaks the symmetry between them.

Fig. 5 shows the formation of a several solitons in the presence of damping, from a FCP input which is a bit less short than previously. We use a super-Gaussian input (namely $U=2 \cos (1.5 T)$ $\left.\exp \left(-(T / 12)^{4}\right)\right)$, and a normalized propagation distance $Z=20$, which would correspond to about $1 \mathrm{~mm}$ for the material parameters discussed above. The general observation is that absorption does not prevent soliton formation. However, the fifth soliton turns into dispersive waves for the highest of the damping values considered (see the bump of the black dash-dotted line on the right of Fig. 5). Recall that the KdV model runs in the frame which moves at the velocity of low-frequency linear waves, corresponding to the index $n$. The dispersive waves run slower, and come at higher times, while the solitons run faster, and arrive at lower times. In fact, in the absence of damping, the fifth soliton, at the beginning of the process, is only a bump of dispersive waves: its velocity is less than $c / n$. Then it receives energy from nonlinear interaction with dispersive waves coming from the left of the figure and running faster to the right. When it has reached the adequate energy level, it becomes a soliton, and its speed becomes higher than $c / n$, i.e., it starts going toward the left of the figure. If damping is stronger, this process cannot occur, mainly because the dispersive waves loose their energy, and consequently the soliton cannot be formed. In other words, if a soliton is present in the input in the sense of the inverse scattering transform, but not visibly present as a large and narrow hump, its formation may be prevented by a strong enough damping, which is not the case for the solitons which propagate without requiring a so strong reshaping.

\section{Conclusion}

Using an analysis based on the Maxwell equation and an assumption in which damping effect is considered as a perturbation, we have shown that the Korteweg-de Vries-Burgers' equation is a partial differential equation which describes the dynamics of $(1+1)$ dimensional few-optical-cycle damped solitons propagation, without using the slowly varying envelope approximation. Analytical and numerical solution of the KdVB equation shows that by considering damping effect, both the amplitude and propagation speed of the solitary wave decrease while the corresponding width of the pulse increases during the propagation distance. It has been shown that damping is completely negligible for transparent materials, and that soliton damping arises in materials which already are considered as appreciably absorbing. Numerical investigation show that, even in highly absorbing media, the linear absorption does not prevent soliton formation, except that the number of solitons may be reduced when it is quite large. The present theory does not take into account guiding properties of the medium, which may influence appreciably the nonlinear propagation. This delicate problem is left for further investigation.

\section{Acknowledgment}

This work was supported by Shahid Beheshti University.

\section{References}

[1] T. Brabec, F. Krausz, Reviews of Modern Physics 72 (2000) 545.

[2] R. Ell, et al., Optics Letters 26 (2001) 373.

[3] D.H. Sutter, et al., Applied Physics B: Lasers and Optics 70 (2000) S5.

[4] J. Bonse, S. Baudach, J. Krüger, W. Kautek, M. Lenzner, Applied Physics A: Materials Science \& Processing 74 (2002) 19.

[5] A. Scrinzi, M.Yu. Ivanov, R. Kienberger, D.M. Villeneuve, Journal of Physics B: Atomic, Molecular and Optical Physics 39 (2006) R1.

[6] F. Krausz, M. Ivanov, Reviews of Modern Physics 81 (2009) 163.

[7] A. Kumar, V. Mishra, Physical Review A: Atomic, Molecular, and Optical Physics 79 (2009) 063807.

[8] T. Brabec, F. Krausz, Physical Review Letters 78 (1997) 3282.

[10] A.A. Voronin, A.M. Zheltikov, Physical Review A: Atomic, Molecular, and Optical Physics 78 (2008) 063834.

[11] Q. Lin, E. Wintner, Optics Communication 150 (1998) 185.

[12] H. Leblond, I.V. Mel'nikov, D. Mihalache, Physical Review A: Atomic, Molecular, and Optical Physics 78 (2008) 043802.

[13] I.V. Mel'nikov, D. Mihalache, F. Moldoveanu, N.-C. Panoiu, Physical Review A: Atomic, Molecular, and Optical Physics 56 (1997) 1569, JETP Letters 65 (1997) 393.

[14] H. Triki, H. Leblond, D. Mihalache, Optics Communication 285 (2012) 3179.

[15] H. Leblond, F. Sanchez, Physical Review A: Atomic, Molecular, and Optical Physics 67 (2003) 013804.

[16] H. Leblond, S.V. Sazonov, I.V. Mel'nikov, D. Mihalache, F. Sanchez, Physical Review A: Atomic, Molecular, and Optical Physics 74 (2006) 063815.

[17] A.V. Kim, S.A. Skobelev, Physical Review A: Atomic, Molecular, and Optical Physics 83 (2011) 063832.

[18] H. Leblond, H. Triki, D. Mihalache, Physical Review A: Atomic, Molecular, and Optical Physics 84 (2011) 023833.

[19] H. Leblond, H. Triki, F. Sanchez, D. Mihalache, Optics Communication 285 (2012) 356.

[20] N.N. Rosanov, V.E. Semenov, N.V. Vysotina, Quantum Electronics 38 (2008) 137.

[21] H. Leblond, D. Mihalache, Journal of Physics A: Mathematical and Theoretical 43 (2010) 375205.

[22] E.D. Farnum, J.N. Kutz, Optics Letters 35 (2010) 3033.

[23] E.V. Kazantseva, A.I. Maimistov, Physics Letters A 263 (1999) 434. 
[24] X. Song, W. Yang, Z. Zeng, R. Li, Z. Xu, Physical Review A: Atomic, Molecular, and Optical Physics 82 (2010) 053821.

[25] H. Leblond, Physical Review A: Atomic, Molecular, and Optical Physics 78 (2008) 013807.

[26] H. Leblond, H. Triki, D. Mihalache, Physical Review A: Atomic, Molecular, and Optical Physics 85 (2012) 053826.

[27] S. Scandolo, F. Bassani, Physical Review B 51 (1995) 6925.

[28] S. Scandolo, F. Bassani, Physical Review B 51 (1995) 6928.

[29] T. Taniuti, C.-C. Wei, Journal of the Physical Society of Japan 24 (1968) 941.

[30] H. Leblond, Journal of Physics B: Atomic, Molecular and Optical Physics 41 (2008) 043001.

[31] C.S. Gardner, J.M. Greene, M.D. Kruskal, R.M. Miura, Physical Review Letters 19 (1967) 1095.
[32] R. Hirota, Solitons, in: R.K. Bulough, P.J. Caudrey (Eds.), Springer, Berlin, 1980 , p. 157 , Chap. 5 .

[33] J. Boussinesq, Journal de Mathématiques Pures et Appliquées 17 (1872) 55

[34] R.W. Boyd, Nonlinear Optics, 3rd ed., Academic Press, San Diego, 2007.

[35]〈http://www.redoptronics.com/BBO-crystal.html 〉.

[36] 〈http://www.eksmaoptics.com/repository/catalogue/pdfai/Pro duct\%20Catalogues/Crystals.pdf $\rangle$.

[37] T.-J. Chen, R.N. Zitter, R. Tao, Physical Review A: Atomic, Molecular, and Optical Physics 51 (1995) 706.

[38] E. Ott, R.N. Sudan, Physics of Fluids 13 (1970) 1432.

[39] E. Ott, R.N. Sudan, Physics of Fluids 12 (1969) 2388

[40] S.M. Cox, P.C. Matthews, Journal of Computational Physics 176 (2002) 430. 